\title{
The Research of Train Energy-Efficient Operation Strategy Based on Multi-Objective Optimization
}

\author{
Yunzhen $\mathrm{Luo}^{1}$ and Mi An ${ }^{2}$ \\ ${ }^{1}$ School of Electronics and Information Engineering, Beijing Jiaotong University, Beijing 100044 \\ ${ }^{2}$ Center for Intelligent Systems and Renewable Energy, Beijing Jiaotong University, Beijing 100044
}

\begin{abstract}
In order to reducing the energy consumption of the train running between the stations, ensuring punctuality and the comfort of the passengers, this paper studies the train energyefficient operation strategy. After taking account of the slope and the speed limit of the line, the model of multi-objective optimization train energy-efficient is established based on train energy consumption, running time and passenger comfort. The improved multi-objective genetic algorithm (MOGA) is used to optimize the target speed sequence to obtain the operation strategy of the train. Different from previous multi-objective optimization, the energy-efficient driving optimization method is realized by considering automatic train operation's (ATO) double-level control structure, slope equivalent strategy, and Pareto optimization in this paper. Based on the actual line data and vehicle parameters of Yizhuang line in Beijing subway, the optimization method is verified by simulation. The simulation results show that, after using the improved multi-objective genetic algorithm, the energy consumption and running time of the train in Yizhuang train station are obviously decreased, and after the train comfort is measured, the rate of change in acceleration or deceleration meet the requirements of passenger experience needs. It can be seen that the proposed algorithm can effectively reduce the energy consumption of the train, ensure the accuracy of the running time and improve the comfort of the passengers.
\end{abstract}

Keywords-Multi-Objective Genetic Algorithm (MOGA); ATO (Automatic Train Operation); ATO double-level

\section{INTRODUCTION}

Because of the rising energy prices and environmental concerns the energy efficiency of transportation systems become more and more important[1]. The main form of energy consumption during the operation of urban rail transit is electricity consumption. Among them, the majority of energy consumption is traction power supply system, accounting for $60 \%-70 \%$ of the total railway energy consumption. Therefore, if we want to reduce the cost of train operations the key way is to reduce the traction energy consumption. As an important subsystem in the automatic train control system (ATC), automatic train operation (ATO) subsystem is mainly used to realize the automatic operation control of the train according to the constraint of inter-station. Therefore, an important way to reduce the energy consumption of the urban rail transit is through the ATO system to control the train. Then use energyefficient optimization algorithm to obtain recommended speed profile. Finally use driving strategy to guide the train running tracing the recommended speed profile automatically, thereby reducing the energy consumption during the train operation.

Ichikawa was the earliest researcher who studied and designed the train driving strategy in the 1960s[2]. In 1980, a train control system was analyzed and designed by Milroy in his doctoral thesis where he studied the train operation strategy without slope or small slope. After that, by the use of the pontryagin maximum principle he get the general form of optimal control for each section[3]. Khmelnitsky established a continuous type of energy-efficient control model, using the pontryagin maximum principle analysis and proved that the train's optimal driving strategy was consisted by four phase: maximum traction, cruising, coasting, the maximum brake[4]. Golovitcher used the maximum principle to obtain the speed profile and the optimal condition transition point sequence equation, furthermore the numerical solution was analyzed[5]. After that a simulation was carried out by Liu et al. according to the different train types[6]. Lu et al. Proposed a distancebased train speed profile search model, and used ant colony algorithm, genetic algorithm and dynamic programming to find the optimal speed profile[7]. Chuang et al. using the artificial neural network to make optimize got the optimal coasting point, then ensure that the train can achieve the minimum operating energy consumption and running time[8]. Yinping $\mathrm{Fu}$ calculated the train energy-efficient control mode conversion point position combined with a specific operating sequence table [9]. Ke et al. using the idea of discrete combination optimization, made the train energy-efficient optimization problem discretized and then used ant colony optimization algorithm to make combinatorial optimization[10, 11]. Xuesong Yu improved the algorithm to solve the problem, and further improved the calculation time[12]. Li et al. after considering the energy consumption, train running time and other objectives, put forward a multi-objective train scheduling model, then developed a timetable for train energy- efficient [13]. In 2013, Shuai Su et al. used the numerical algorithm to distribute the train running time, and then reduce the train energy consumption between the two stations by optimizing the speed profile[14].On the basis of multi-objective optimization method and theory, Jin Yu builds up the train running process optimization model with consideration of train punctuality, train parking accuracy and the train energy consumption. Finally, the improved particle swarm algorithm is used to solve the problem[15]. In 2014, Fengchu Long designed an improved genetic algorithm to calculate the inter-station coast control point in time constraints and take full account of the needs of passenger comfort and train running time[16]. María 
Domínguez et al. Used the multi-objective particle swarm optimization algorithm to optimize the speed profile of the train, taking account of the energy consumption of the train, the running time and the passenger comfort[17].

In summary, domestic and foreign scholars have made a wide range of research in the train energy-efficient operation model, algorithms and simulation methods. Totally they only just from the aspect of use different optimization algorithms or the aspect of improve the optimal algorithms, without consider the train ATO control structure. As FIGURE I shows, this paper considering both the previous study and the double-level control structure of ATO. The high-level control is used to get a recommended speed profile satisfying the optimization objective under the condition of the sensor data, line data, vehicle data and timetable. The optimization objectives usually consist of comfort, punctuality and energy efficiency, accurate stop etc. By making a comparison between the recommended speed and the practical speed, the low-level outputs the corresponding control instructions to traction or braking unit to track the recommended speed profile.

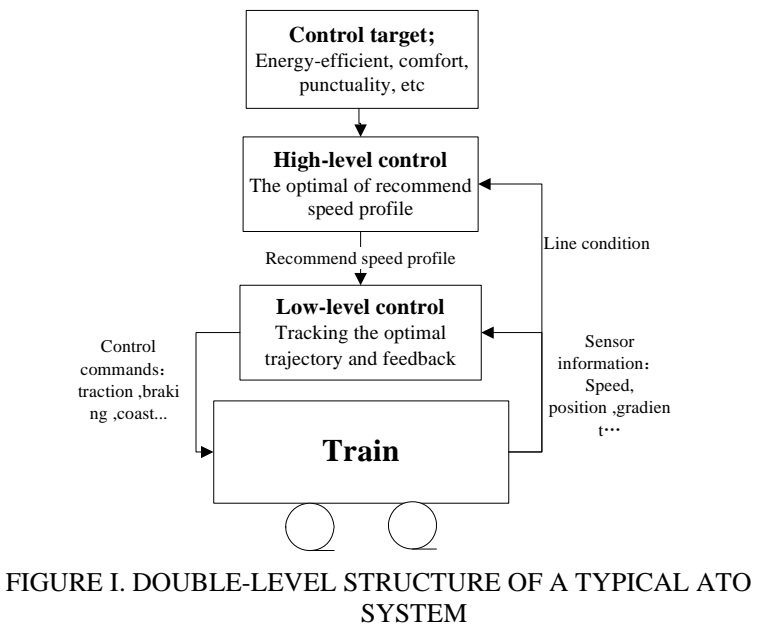

The main contribution of this paper is described as follows. First of all this paper proposed an improved optimization method for train recommended speed profile based on MOGA which has the elitism realized by non-dominant sorting strategy. The MOGA is improved through the use of immigration operator, which makes the different control parameters of the GA algorithm linked. It's useful to the selection of the elite populations for it doesn't let the elite populations carry out the corresponding selection, crossover, mutation and other genetic operations. By use this method, it can avoid destroying the optimal individuals and make full use of the advantages of knowledge accumulation in the evolution process of GA. Secondly this paper also considering the ATO double-level control structure. The high-level calculate a recommend speed profile, the low-level tracking the trajectory and make a feedback to high level. In addition, the train's energy consumption and running time are further reduced through decreasing the switching frequency of traction during cruising phase. Furthermore, the convergence speed of this improved algorithm is fast, an effective recommended speed profile can be with optimization fasted. Finally, Based on the actual line data and vehicle parameters of Yizhuang line in Beijing subway, the optimization method is verified by simulation. The results demonstrate the reliability and effectiveness of this energy-efficient method, which has a very important practical significance.

The rest of this paper is organized as follows. In part II, we described the problem with train motion model and multiobjective model. Furthermore we present the method of optimization and considered ATO tracking strategy in the process of optimization. In part III, the optimizing algorithms was presented for recommended speed profile with considering Pareto optimization, ATO tracing strategy and slope equivalent strategy in the process of optimization. In part IV a case study is presented to indicate the performance of proposed algorithm.

\section{PROBLEM DESCRIPTION AND MODEL}

The problem is described as to find out the best energyefficient recommended speed profile under considering the punctuality of the train and the comfort of the passengers. More specifically, it's a multi-objective optimization problem. Usually, the goals in the multi-objective optimization problem are conflicting and interacting, and when we optimize one of the sub-goals to make them better, they often create other subobjective Performance degradation. Therefore, when solving the multi-objective optimization problem, there is no absolute optimal solutions that can make all the sub-objective reach the optimal, but we can get a relative sense of better solution and the most appropriate solution according to the requirements of real life.

As show in FIGURE II, the different recommended speed profile will result different energy consumption. Therefore the optimization problem is transformed into recommended speed profile optimization with minimum energy consumption, train punctuality and passengers comfort. As shown in the FIGURE II, the rail line between two adjacent stations (station A and B) is discretized into $\mathrm{N}$ segment by the characteristic of the line (see equal 2). After the line discretized, the recommended speed $\mathrm{V}$ selected in every discrete segment is discretizing equally in $\mathbf{M}$ part (see equal 3). Based on the above analysis, the optimization function of energy is:

$$
\begin{gathered}
E(x)=\min \sum_{i=1}^{N} E\left(x_{i}, v_{i}\right) \\
x \in\left\{x_{1}, x_{2}, x_{3}, \ldots, x_{N-1}, x_{N}\right\}, s=\sum_{i=1}^{N} x_{i} \\
v \in\left\{v_{1}, v_{2}, v_{3}, \ldots, v_{M-1}, v_{M}\right\}
\end{gathered}
$$

Where $E(x)$ is the energy consumption after finished all choices of speed codes at every discrete point, $E\left(x_{i}, v_{i}\right)$ is the energy consumption of point ${ }^{X_{i}}$ where the recommended speed 
is $v_{i}, \mathrm{~N}$ and $\mathrm{M}$ is the number of discrete point, $\mathrm{s}$ is the distance between station A to station $\mathrm{B}$.

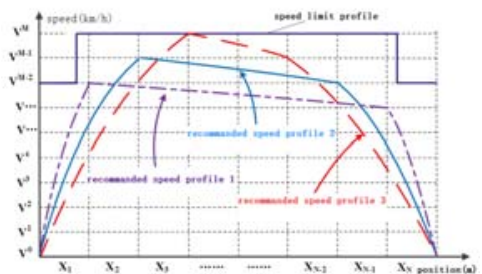

FIGURE II. THE RECOMMENDED SPEED PROFILE OPTIMIZATION

\section{A. The Train Motion Equations}

$$
\left\{\begin{array}{l}
v \frac{d v}{d x}=F_{p}(p, v)-f_{b}(b, v)-f_{o}(v)-f_{a d d}(x) \\
\frac{d t}{d x}=\frac{1}{v}
\end{array}\right.
$$

$$
f_{\text {add }}(x)=f_{g}+f_{c}+f_{t}
$$

Where $x$ is the position of the train, $v(x)$ is the speed of the train at position $x, F_{p}(p, v)$ is the specific maximum traction force per mass unit, $f_{b}(b, v)$ is the specific maximum braking force per mass unit, $f_{o}(v)$ is the specific maximum braking force per mass unit, $f_{a d d}(x)$ is the specific add resistance cased by gradient, curve and tunnel.

\section{B. The Multi-Objective Model}

$$
\begin{gathered}
\min Y=F(x)=\left(E(x), T_{\text {error }}(x), \operatorname{Jerk}(x)\right)^{T} \\
E(x)=E_{t}+E_{c}+E_{b} \\
T_{\text {error }}(x)=e^{\frac{\left|T-T_{a}\right|-t_{a}}{2 \sigma^{2}}} \\
\operatorname{Jerk}(x)=e^{\frac{\mid \frac{d_{a}}{d_{t}}-a_{k}}{2 \sigma^{2}}}
\end{gathered}
$$

Where the final value of $F(x)$ is the whole metrics for the objective of energy consumption, punctuality and comfort. $E(x)$ is the membership function of energy, $E_{b}$ is the energy consumption caused by braking, $E_{t}$ is the energy consumption caused by traction, $E_{c}$ is the energy consumption caused by cruising; $T_{\text {error }}(x)$ is the membership function of punctuality, ${ }^{t}$ is the acceptable running time error, $T$ is the full plan running time of the train, ${ }^{T_{a}}$ is the actual running time of the train; $\operatorname{Jerk}(x)$ is the membership function of comfort, $a_{k}$ is the target comfort value.

\section{The Tracking Strategy of ATO}

It was proved to be the most energy efficient way that after the line was discretized, the train should have the maximum traction at first segment, and have the maximum braking at last segment [18]. When the train runs on other segments, if the difference between recommended speed and tracked speed is less than $2 \mathrm{~km} / \mathrm{h}$, the traction phase will be switched to coasting phase to preventing the train speed exceed the limit speed. When the difference between recommended speed and tracked speed is more than $3 \mathrm{~km} / \mathrm{h}$, the coasting phase will be switched to traction phase to assure the train of runs at a high speed to decrease the running time.

\section{SOLUTIONS AND INFRASTRUCTURE}

\section{A. Multiple Population Genetic Algorithm (MPGA)}

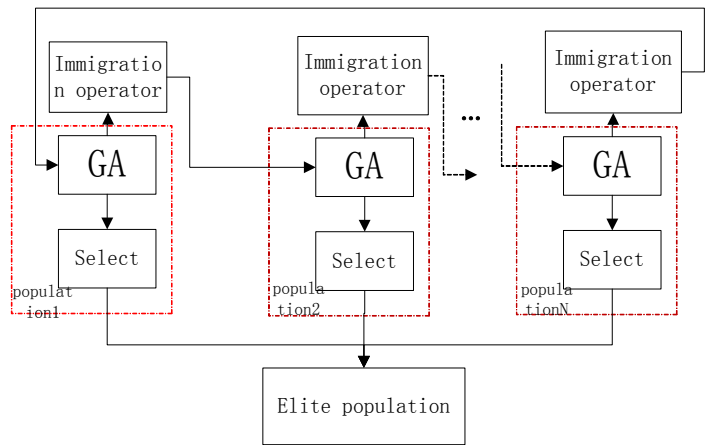

FIGURE III. MULTIPLE POPULATION GENETIC ALGORITHM

A key contribution of this paper is described as follows. First this paper proposed an improved optimization method for recommend speed profile based on MOGA which has the elitism and non-dominant sorting strategy.

As we can see in the fig.3, compared with GA, the MPGA have some advantages like follows:

(1) The limitation of relies on a single population for evolution of conventional genetic algorithm can be broken In order to achieve different search purposes, the algorithm introduced multiple populations to search at the same times, and with each group is given different control parameters.

(2) In order to achieve multi-population co-evolution, the algorithm is communicated through the immigration operator. The immigration operator will introduce the optimal 
individuals from this population into another population, allowing the exchange of information between the populations. By use this method it can avoid the algorithm get into a stagnant state. Finally, the optimal individuals in each population are placed in the elite population by selecting the operator manually.

\section{B. Slope Equivalent Strategy}

Calculate the average slope of the segment according to the average of the three-point slope: the train front, middle, and rear. The specific content is: the train with the length of $l$ is discretized into several segments equally; during the train running, the slope equivalent strategy will make all the slope of discrete point equal to $G(s)$ use the following formula.

$$
G(s)=\left\{\begin{array}{l}
\frac{\sum_{i=1}^{s} g_{i}}{s}, s<l \\
\frac{\sum_{i=s-l+1}^{s} g_{i}}{l}, s \geq l
\end{array}\right.
$$

Where $S$ is the position of the train in the running line, $l$ is the length of the train, $g_{i}$ is the slope of the train when the train is at the discrete point, $G(s)$ is the equivalent slope of the train.

\section{Algorithm Process}

The schematic of the energy-efficient method for optimization of the recommended speed profile is shown in FIGURE V. As we can see, first the interstation distance is divided by a given number of segments by the line data and slope data to construct the discrete combination optimization problem. Then with the proposed algorithm which considered the ATO double-level and elite strategy, the optimal recommended speed profile can be got and tracked well. It is worth mentioning that the elite population can be obtained by operation like FIGURE III. Otherwise we set a maximum iteration I to assure that we get a best result under the acceptable time. It's well described in part III-B for the slope equivalent strategy and how to get the elite population in part III-A.

As for the selection strategy used in this paper is roulette Wheel Selection, so that can guarantee the individual with greater fitness value will have the greater probability be selected into next generation in the process of evolution. The current crossover method include Single-point, doublepoint and shuffle crossover. This paper used double-point crossover, if the two individuals that used to crossover are same, we will make one of them be mutated for get a more fast convergence speed of this algorithm.

The fitness function is the standard of individual quality, the value of individual's fitness decided whether it can be choose into next generation. In addition, it is specifies that the fitness function must be positive, and the more big of the fitness function value, the more that the result can be closer to the optimal solution. For that the original objective function is the minimum problem, it is necessary to make a conversion. Therefore the formula of (7) will be converted to its reciprocal. But as for the fitness function of time, from FIGURE IV we can see that if the value of $\left|T-T_{a}\right|$ is between $-t_{a}$ to $t_{a}$, the value of $T_{\text {error }}(x)$ is zero (in fig. $4\left|T-T_{a}\right|=x, t_{a}=4, \sigma=0.009$ ), if not, the individual will not be selected to next generation. Therefore the fitness functions of time and functions of comfort can keep the same function.

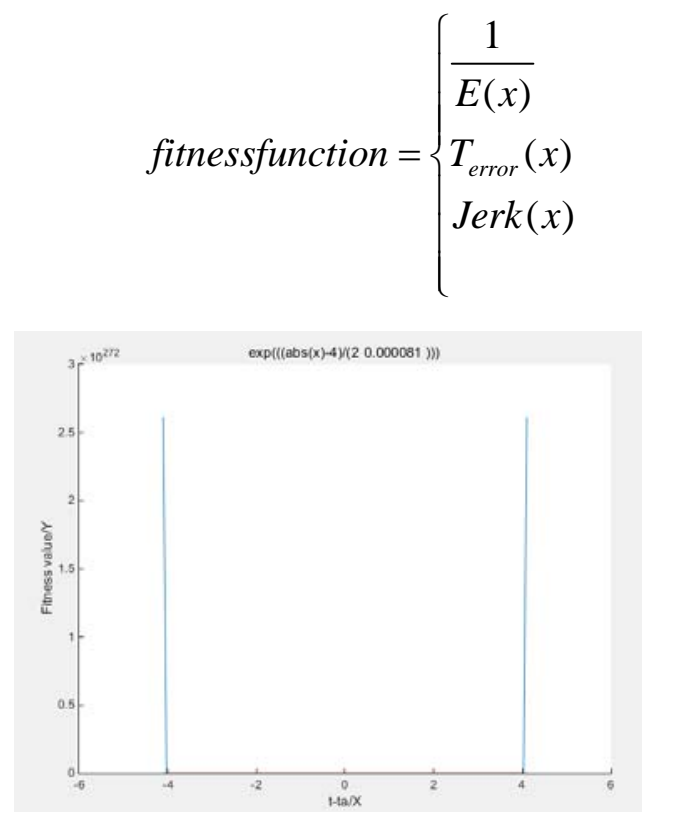

FIGURE IV. THE FITNESS FUNCTION OF TRAIN RUNNING TIME 


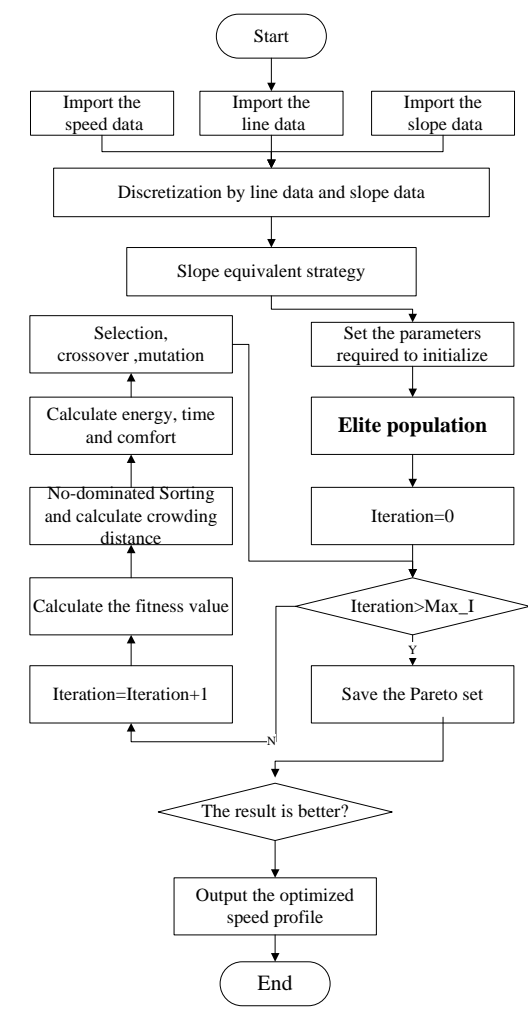

FIGURE V. THE SCHEMATIC OF THE ENERGY-SAVING METHOD FOR OPTIMIZATION OF THE RECOMMENDED SPEED PROFILE

IV.

SIMULATION AND ANALYSIS

\section{A. Operation Data}

In the condition of no-load train (AW0), the traction/braking characteristic curve of the train in Beijing subway Yizhuang line are described like follows:

The maximum traction force $(\mathrm{KN})$ :

$$
F_{\text {Max }}=\left\{\begin{array}{l}
203 ; 0 \leq v \leq 51.5 \\
-0.002032 v^{3}+0.4928 v^{2}-42.12 v+1343 \\
51.5 \leq v \leq 80
\end{array}\right.
$$

The maximum braking force $(\mathrm{KN})$ :

$$
B_{\text {Max }}=\left\{\begin{array}{l}
166 ; 0 \leq v \leq 77 \\
0.134 v^{2}-25.07 v+1300 \\
77 \leq v \leq 80
\end{array}\right.
$$

In the above formula, $v$ is the running speed of the train, the unit is $\mathrm{km} / \mathrm{h}, F_{\text {Max }}$ is the maximum traction force, the unit is $\mathrm{KN}, B_{\text {Max }}$ is the maximum braking force, the unit is $\mathrm{KN}$.

\section{B. Line Data}

Select Yizhuang train station to sub-station from Yizhuang line for the operation of the example, the distance between the two stations is 1334 meters, the train running time is 110 seconds from the timetable, and the station stop time is 45 seconds. The traction energy consumption from TMS (Train Management System) is $15.67 \mathrm{kWh}$ without use our algorithm. The following table $\mathrm{I}$, is the line slope information of the operating range. The limit speed of the station is $80 \mathrm{~km} / \mathrm{h}$ from $0 \mathrm{~m}$ to $1214 \mathrm{~m}$, and the maximum speed of the train in the rest of the line is $55 \mathrm{~km} / \mathrm{h}$.

\section{TABLE I. THE GRADIENT DATA FROM YIZHUANG TO CIQU}

\begin{tabular}{|c|c|c|c|}
\hline Segment & $0-193$ & $193-533$ & $533-928$ \\
\hline gradient & 2 & -19.7 & 3.133 \\
\hline Segment & $\begin{array}{c}928- \\
1128\end{array}$ & $1128-1334$ & \\
\hline gradient & 20 & 2 & \\
\hline
\end{tabular}

C. The Train Parameters

TABLE II. THE TRAIN PARAMETERS OF BEIJING SUBWAY

\begin{tabular}{|l|l|}
\hline Train parameters & value \\
\hline Train type & type B \\
\hline Train formation & 194.295 (AW0) \\
\hline Train weight(ton) & 140 \\
\hline Train length(ton) resistance & 2.031 \\
\hline Maximum train speed(km/h) & 0.0622 \\
\hline $\begin{array}{l}\text { Train basic resistance } \\
\text { coefficient A basic deceleration }\end{array}$ & 1 \\
\hline $\begin{array}{l}\text { Train } \\
\text { coefficient B }\end{array}$ & 0.001807 \\
\hline $\begin{array}{l}\text { Train basic resince } \\
\text { coefficient C }\end{array}$ & 1 \\
\hline $\begin{array}{l}\text { Maximum } \\
\left(m / s^{2}\right)\end{array}$ & $\begin{array}{l}\text { Maximum } \\
\left(m / s^{2}\right)\end{array}$ \\
\hline
\end{tabular}

\section{Case Study}

The algorithm is verified by the simulation which used the data of Yizhuang line. The simulation process is like follows:

Case 1: the genetic algorithm is applied to get the recommended speed profile without use the elitism nondominant sorting strategy and without considering the slope equivalent strategy.

The recommended speed profile based on the running time of timetable after optimization is show in FIGURE VI. 


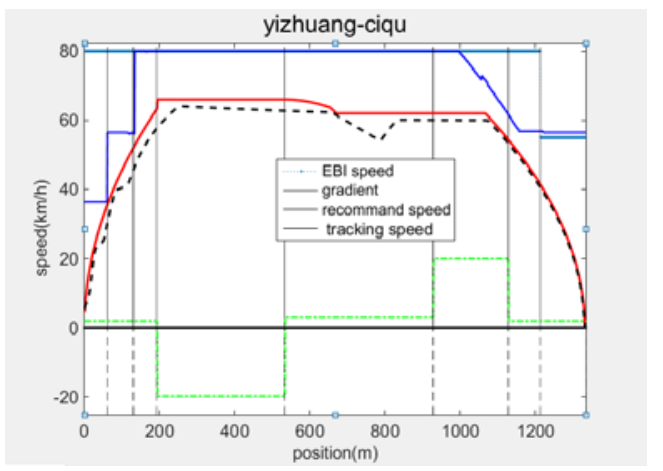

FIGURE VI.THE RECOMMENDED SPEED PROFILE OF CASE 1

Case 2: the MPGA algorithm is applied in order to get the optimal recommended speed profile with considering Pareto optimization, ATO tracing strategy and slope equivalent strategy.

The recommended speed profile based on the running time of timetable after optimization is show in FIGURE VII.

The Pareto front with considering train energy consumption and train running time is showed in FIGURE VII.

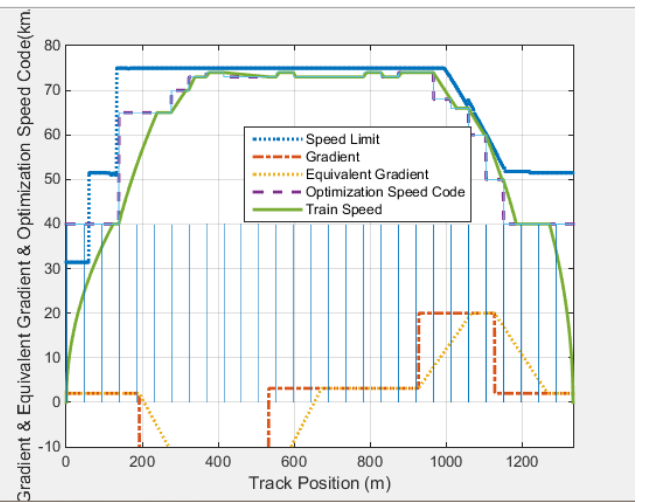

FIGURE VII. THE RECOMMENDED SPEED PROFILE OF CASE 2

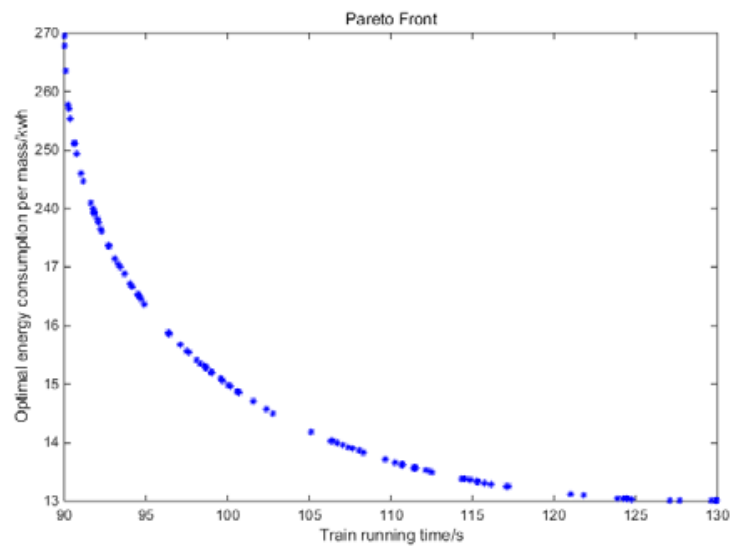

FIGURE VIII. THE PARETO FRONT WITH CONSIDERING TRAIN ENERGY CONSUMPTION AND TRAIN RUNNING TIME OF CASE 2

The comparison result is showed in follow table:
TABLE III. THE COMPARISON RESULT OF CASE 1 AND CASE 2

\begin{tabular}{|c|c|c|}
\hline Parameters & Case 1 & Case 2 \\
\hline Schedule time & 110 & 110 \\
\hline Running time & 107.56 & 111.76 \\
\hline $\begin{array}{c}\text { Energy } \\
\text { consumption }(\mathrm{kw} / \mathrm{h})\end{array}$ & 14.32 & 13.98 \\
\hline Energy efficiency $(\%)$ & 8.61 & 10.78 \\
\hline
\end{tabular}

As the TABLE III shows that the running time of both case 1 and case 2 meets the requirement of timetable. But the recommended speed profile is well optimized in case 2, decreased energy consumption to $1.69 \mathrm{kw} / \mathrm{h}$. the optimal recommended speed profile can save energy-consumption up to $10.78 \%$ with almost the same running time and the same comfort. It indicates that the proposed algorithm has a well performance on energy-saving train operation.

\section{CONCLUSION}

In this paper, a multi-objective genetic algorithm is used as the core method, and a multi-objective train energy-efficient optimization model based on double-level control structure is proposed. Firstly the rail line between two adjacent stations is discretized into $\mathrm{N}$ segments by the characteristics of the line and the recommended speed $\mathrm{V}$ selected in every discrete segment which is discretized equally; secondly, the slope equivalent method will be used. After that the multi- objective genetic algorithm is used to obtain the recommended speed profile through Pareto optimization. Finally, the tracing strategy is used to get the real train running speed profile. The simulation results show that the proposed algorithm is more energy efficient and more punctuality than the simple genetic algorithm. The case study based on the data of Yizhuang station to Ciqu station verified the feasibility and effectiveness of the proposed method on train energy-efficient operation. Moreover the optimal recommended speed profile can save energy-consumption up to $10.78 \%$ with almost the same running time and the same comfort.

This paper only considers the energy-efficient operation of a single train, does not take account of multiple trains running on the same line at the same time. Multi-train tracking energyefficient operation is the next step in the further research.

\section{REFERENCES}

[1] Hansen, I.A., Railway timetable \& traffic: analysis, modelling, simulation. 2008: Eurailpress.

[2] Ichikawa, K., Application of optimization theory for bounded state variable problems to the operation of train. Bulletin of JSME, 1968. 11(47): p. 857-865.

[3] Milroy, I.P., Aspects of automatic train control. 1980, (c) Ian Peter Milroy.

[4] Khmelnitsky, E., On an optimal control problem of train operation. IEEE Transactions on Automatic Control, 2000. 45(7): p. 1257-1266.

[5] Golovitcher, I.M. Energy efficient control of rail vehicles. in Systems, Man, and Cybernetics, 2001 IEEE International Conference on. 2001. IEEE.

[6] Liu, R.R. and I.M. Golovitcher, Energy-efficient operation of rail vehicles. Transportation Research Part A: Policy and Practice, 2003. 37(10): p. 917-932.

[7] Lu, S., et al., Single-train trajectory optimization. IEEE Transactions on Intelligent Transportation Systems, 2013. 14(2): p. 743-750. 
[8] Chuang, H.-J., et al. Design of optimal coasting speed for saving social cost in mass rapid transit systems. in Electric Utility Deregulation and Restructuring and Power Technologies, 2008. DRPT 2008. Third International Conference on. 2008. IEEE.

[9] Yinping F U, Ziyou G A O, Keping L. Modeling study for tracking operation of subway trains based on cellular automata[J]. Journal of Transportation Systems Engineering and Information Technology, 2008, 8(4): 89-95.

[10] Ke, B.-R., M.-C. Chen, and C.-L. Lin, Block-layout design using MAXMIN ant system for saving energy on mass rapid transit systems. IEEE Transactions on Intelligent Transportation Systems, 2009. 10(2): p. 226235.

[11] Ke, B.-R., C.-L. Lin, and C.-W. Lai, Optimization of train-speed trajectory and control for mass rapid transit systems. Control Engineering Practice, 2011. 19(7): p. 675-687.

[12] Xuesong Yu, Urban Rail Transit Train Energy Optimization and Energy Consumption Assessment. 2012, Beijing Jiaotong University.

[13] Li, X., et al., A green train scheduling model and fuzzy multi-objective optimization algorithm. Applied Mathematical Modelling, 2013. 37(4): p. 2063-2073.

[14] Su, S., et al., A subway train timetable optimization approach based on energy-efficient operation strategy. IEEE Transactions on Intelligent Transportation Systems, 2013. 14(2): p. 883-893.

[15] Jin Yu, Multi-target Train Operation Process Optimization and Control Strategy Research, 2009, Southwest Jiaotong University.

[16] Feng chu Long, Optimization of optimal laziness point of train based on genetic algorithm. 2014, Beijing Jiaotong University.

[17] Domínguez, M., et al., Multi objective particle swarm optimization algorithm for the design of efficient ATO speed profiles in metro lines. Engineering Applications of Artificial Intelligence, 2014. 29: p. 43-53.

[18] Howlett, P.G., P.J. Pudney, and X. Vu, Local energy minimization in optimal train control. Automatica, 2009. 45(11): p. 2692-2698. 